\title{
SINGULARITIES OF NULL MEAN CURVATURE FLOW OF NULL HYPERSURFACES IN LORENTZIAN MANIFOLDS
}

\author{
SAMUEL SSEKAJJA ${ }^{1}$, FORTUNÉ MASSAMBA ${ }^{2}$
}

\begin{abstract}
We classify two main singularities, as type I and type II, associated to null mean curvature flow of screen conformal null hypersurfaces in Lorentzian manifolds. We prove that the flow at a type I singularity is asymptotically self-similar, whereas at a type II singularity there is a blow-up solution which is an eternal solution. For further analysis of the above two singularities, we define null translating solitons and use them to prove some Harnack estimates for null mean curvature flow under certain geometric conditions.
\end{abstract}

\section{Introduction}

The theory of submanifolds of a Riemannian (or semi-Riemannian) manifold is one of the most important topics of differential geometry (see for example, O'Neill [19]). While the geometry of semi-Riemannian submanifolds is fully developed, its counter part of null (lightlike, degenerate) submanifolds (for which the local geometry is completely different than the non-degenerate case) is relatively new and in a developing stage (see $[4,5$, $7,8,11,17,22]$ and others referred therein). The theory has numerous applications in mathematical physics, particularly in general relativity and electromagnetism (see [7]). In general relativity, null hypersurfaces can be viewed as models of different black hole horizons (see $[1,3,7,8]$ ).

In [22], the authors introduced the concept of evolving null hypersurfaces by their null mean curvature, in which several aspects of such hypersurfaces were investigated. It was shown, under certain geometric conditions-such as screen conformality [7], that null mean curvature flow also develops singularities. Here we want to study the singularities of associated to null mean curvature flow which can occur for non convex initial data. Our aim is to

2010 Mathematics Subject Classification. Primary 53C25; Secondary 53C40, 53C50.

Key words and phrases. Null hypersurfaces; Null mean curvature flow; Singularities; Harnack estimates. 
characterize the asymptotic behavior of such sufaces near a singularity using rescaling techniques. These methods have been used in the study of singularities arising from the classical mean curvature flow of hypersurfaces in Riemannian geometry $[13,14]$. For more details on the classical mean curvature flow we refer to $[2,6,10,9,12,13,14,15,16,18,20,21]$ and many more references therein.

The paper is arranged as follows; In Section 2, we review the basic concepts on null hypersurfaces. We also recall some known results on null mean curvature flow required in the rest of the paper. In Section 3, we derive a null monotonicity formula which we then use to investigate singularities in null mean curvature flow. Finally, in Section 4, we prove a set of Harnack estimates for null mean curvature flow.

\section{Preliminaries}

Let $(\bar{M}, \bar{g})$ be a $(n+2)$-dimensional Lorentzian manifold with index $q \in$ $\{1, \ldots, n+1\}$ and let $M$ be a hypersurface of $\bar{M}$. Denote by $g$ the induced tensor field by $\bar{g}$ on $M$. Then, $M$ is called a null hypersurface of $\bar{M}$ if $g$ is of constant rank $n$. Next, consider the vector bundle $T M^{\perp}$ whose fibers are defined as $T_{x} M^{\perp}=\left\{Y_{x} \in T_{x} \bar{M}: \bar{g}_{x}\left(X_{x}, Y_{x}\right)=0, \forall X_{x} \in T_{x} M\right\}$, for any $x \in M$. Therefore, a hypersurface $M$ of $\bar{M}$ is null if and only if $T M^{\perp}$ is a distribution of rank 1 on $M$. Let $M$ be a null hypersurface, we consider the complementary distribution $S(T M)$ to $T M^{\perp}$ in $T M$, which is called a screen distribution. It is well-known that $S(T M)$ is non-degenerate (see [7]). Thus, we have

$$
T M=S(T M) \perp T M^{\perp} .
$$

As $S(T M)$ is non-degenerate with respect to $\bar{g}$, we have $T \bar{M}=S(T M) \perp$ $S(T M)^{\perp}$, where $S(T M)^{\perp}$ is the complementary vector bundle to $S(T M)$ in $\left.T \bar{M}\right|_{M}$.

Let $(M, g)$ be a null hypersurface of $(\bar{M}, \bar{g})$. Then, there exists a unique vector bundle $\operatorname{tr}(T M)$, called the null transversal bundle [7] of $M$ with respect to $S(T M)$, of rank 1 over $M$ such that for any non-zero section $E$ of $T M^{\perp}$ on a coordinate neighborhood $\mathcal{U} \subset M$, there exists a unique section $N$ of $\operatorname{tr}(T M)$ on $\mathcal{U}$ satisfying $\bar{g}(E, N)=1, \bar{g}(N, N)=\bar{g}(N, Z)=0$, for any section $Z$ of $S(T M)$. Consequently, we have the following decomposition of $T \bar{M}$.

$$
\left.T \bar{M}\right|_{M}=S(T M) \perp\left\{T M^{\perp} \oplus \operatorname{tr}(T M)\right\}=T M \oplus \operatorname{tr}(T M) .
$$


Throughout this paper, $\Gamma(\Xi)$ will denote the $\mathcal{F}(M)$-module of differentiable sections of a vector bundle $\Xi$. Let $\nabla$ and $\nabla^{*}$ denote the induced connections on $M$ and $S(T M)$, respectively, and $P$ be the projection of $T M$ onto $S(T M)$, then the local Gauss-Weingarten equations of $M$ and $S(T M)$ are the following [7]

$$
\begin{aligned}
\bar{\nabla}_{X} Y & =\nabla_{X} Y+h(X, Y)=\nabla_{X} Y+B(X, Y) N \\
\bar{\nabla}_{X} N & =-A_{N} X+\nabla_{X}^{t} N=-A_{N} X+\tau(X) N \\
\nabla_{X} P Y & =\nabla_{X}^{*} P Y+h^{*}(X, P Y)=\nabla_{X}^{*} P Y+C(X, P Y) E, \\
\nabla_{X} E & =-A_{E}^{*} X+\nabla_{X}^{* t} E=-A_{E}^{*} X-\tau(X) E, \quad A_{E}^{*} E=0,
\end{aligned}
$$

for all $X, Y \in \Gamma(T M), E \in \Gamma\left(T M^{\perp}\right)$ and $N \in \Gamma(\operatorname{tr}(T M))$, where $\bar{\nabla}$ is the Levi-Civita connection on $\bar{M}$. In the above setting, $B$ is the local second fundamental form of $M$ and $C$ is the local second fundamental form on $S(T M) . A_{N}$ and $A_{E}^{*}$ are the shape operators on $T M$ and $S(T M)$ respectively, while $\tau$ is a 1 -form on $T M$. The above shape operators are related to their local fundamental forms by

$$
\begin{aligned}
& g\left(A_{E}^{*} X, Y\right)=B(X, Y), \quad g\left(A_{N} X, P Y\right)=C(X, P Y), \\
& \bar{g}\left(A_{E}^{*} X, N\right)=0, \quad \bar{g}\left(A_{N} X, N\right)=0, \quad \forall X, Y \in \Gamma(T M) .
\end{aligned}
$$

From (2.8) we notice that $A_{E}^{*}$ and $A_{N}$ are both screen-valued operators. Let $\vartheta=\bar{g}(N, \cdot)$ be a 1 -form metrically equivalent to $N$ defined on $\bar{M}$. Take $\lambda=i^{*} \vartheta$ to be its restriction on $M$, where $i: M \rightarrow \bar{M}$ is the inclusion map. Then it is easy to show that $\left(\nabla_{X} g\right)(Y, Z)=B(X, Y) \lambda(Z)+B(X, Z) \lambda(Y)$, for all $X, Y, Z \in \Gamma(T M)$, which indicates that $\nabla$ is generally not a metric connection with respect to $g$. However, the induced connection $\nabla^{*}$ on $S(T M)$ is a metric connection. For more details about null hypersurfaces see the books [7] and [8]. Through the 1-form $\lambda$, the authors in [5] considered a non-degenerate metric $\widehat{g}$ on $M$, given by

$$
\widehat{g}(X, Y)=g(X, Y)+\lambda(X) \lambda(Y), \quad \forall X, Y \in \Gamma(T M) .
$$

The metric $\widehat{g}$ is invertible and its inverse, $g^{[\cdot, \cdot]}$, was called the pseudo-inverse of $g$ (see details in [5]). Also, observe that $\widehat{g}$ coincides with $g$ if the latter is non-degenerate. The metric $\widehat{g}$ has been used to define (on $M$ ) the usual operators such as gradient, divergence, D' Alambertian (see [5]), which one can not afford with the degenerate metric $g$. In case $\widehat{g}$ coincide with $g$ on $M$, we define the gradient $\nabla^{s} \varrho$, Hessian, $\operatorname{Hess}^{s}(\varrho)$, and D' Alambertian $\Delta^{s} \varrho$ 
of a smooth function $\varrho$ on $\mathcal{U} \subset M$ with respect to the screen distribution $S(T M)$ as

$$
\begin{aligned}
\nabla^{s} \varrho & =g^{\alpha \beta} X_{\alpha}(\varrho) X_{\beta}, \\
\operatorname{Hess}^{s}(\varrho) & =X_{\alpha}\left(X_{\beta}(\varrho)\right)-\left(\nabla_{X_{\alpha}}^{*} X_{\beta}\right)(\varrho) \\
\text { and } \quad \Delta^{s} \varrho & =\operatorname{tr}^{s}\left(\operatorname{Hess}^{s}(\varrho)\right)=g^{\alpha \beta}\left(X_{\alpha}\left(X_{\beta}(\varrho)\right)-\left(\nabla_{X_{\alpha}}^{*} X_{\beta}\right)(\varrho)\right),
\end{aligned}
$$

where $\left\{X_{1}, \ldots, X_{n}\right\}$ is a basis of $S(T M)$ and $\operatorname{tr}^{s}(\cdot)$ denotes the trace with respect to $S(T M)$. Throughout this paper, we assume that $M$ carries the associated metric $\widehat{g}$ and $\operatorname{tr}(\cdot)$ will denote the trace over $M$ with respect to $\widehat{g}$. In the sequel, we shall make use of the following convention on the range of indices: $1 \leq \alpha, \beta, \gamma, \mu, \sigma \leq n, 0 \leq a, b, c \leq n$ and $0 \leq i, j, k \leq n+1$.

Let $(M, g, S(T M))$ be a 1-null simply connected $(n+1)$-dimensional manifold of index $(q-1)$ and with the geometric objects $\bar{g}, \nabla^{\prime}, \nabla^{t r}, h^{*^{\prime}}$ and $A^{*^{\prime}}$. Then, there exists a null isometric immersion $F: M^{n+1} \longrightarrow \bar{M}^{n+2}$ (see [7, Theorem 4.1] for details) satisfying

$$
g(X, Y)=\bar{g}\left(F_{*} X, F_{*} Y\right), \quad \forall X, Y \in \Gamma(T M),
$$

and a vector bundle isomorphism $\bar{F}: \operatorname{tr}(T M) \longrightarrow \operatorname{tr}(T F(M))$ such that we have $F_{*}\left(\nabla_{X}^{\prime} Y\right)=\nabla_{F_{*} X} F_{*} Y, F_{*}\left(A_{U}^{*^{\prime}} X\right)=A_{F_{*} U}^{*} F_{*} X, F_{*}\left(h^{*^{\prime}}(X, P Y)\right)=$ $h^{*}\left(F_{*} X, F_{*} P Y\right)$ and $\bar{F}\left(\nabla_{X}^{t r} V\right)=\nabla_{X}^{t} \bar{F} V, \forall X, Y \in \Gamma(T M), U \in \Gamma(\operatorname{Rad} T M)$ and $V \in \Gamma(\operatorname{tr}(T M))$, where $\operatorname{tr}(T F(M))$ is the null transversal vector bundle of $F(M)$ with respect to $F_{*} S(T M)$, and $\nabla, \nabla^{t}, h^{*}, A^{*}$ are the geometric objects induced on $F(M)$ with respect to the immersion $F$. Certainly, $F(M)$ is nothing but a 1-null submanifold of $\bar{M}^{n+2}$. Moreover, the immersion $F$ preserves both the radical and screen distribution (see [7] for details).

Next, suppose that $F(M)$ is a null hypersurface as described above. By the method of [7], the null mean curvature vector $\mathrm{H}$ of $F(M)$ at $p \in M$ is a smooth vector field transversal to $F(M)$ and given by

$$
\mathrm{H}=\operatorname{tr}^{s}(h)=\operatorname{tr}^{s}(B) N=\mathcal{S} N,
$$

where $N \in \Gamma(\operatorname{tr}(F T M))$ and $\mathcal{S}:=\operatorname{tr}^{\mathcal{S}}(B)=\operatorname{tr}^{\mathcal{S}}\left(A_{E}^{*}\right)$. The function $\mathcal{S}$ is called the null mean curvature of $F(M)$.

Definition 2.1. [22] Let $\left(M^{n+1}, g, S(T M)\right)$ be a compact null hypersurface of a semi-Riemannian manifold $\left(\bar{M}^{n+2}, \bar{g}\right)$. Assume that $F_{0}: M^{n+1} \times[0, T) \longrightarrow$ $\bar{M}^{n+2}$ smoothly immerses $M$ as a null hypersurface in $\bar{M}$. We say that $M_{0}:=F_{0}(M)$ evolves by its null mean curvature vector if there is a whole 
family $F(\cdot, t)$ of smooth immersions with corresponding hypersurfaces $M_{t}:=$ $F(\cdot, t)(M)$ such that

$$
\frac{\partial F}{\partial t}(p, t)=\mathrm{H}(p, t), \quad F(\cdot, 0)=F_{0}, \quad p \in M^{n+1},
$$

where $\mathrm{H}(p, t):=\mathcal{S}(p, t) N$ is the mean curvature vector of $M_{t}$ at $F(p, t)$.

\section{Singularity formation}

In this section, we discuss the singularities which arises during null mean curvature flow. Let us consider an orthonormal basis $\left\{X_{1}, \ldots, X_{n}\right\}$, where $X_{\alpha}=\frac{\partial}{\partial x_{\alpha}}$, of $S(T M)$ around a point $p \in M$ such that the induced LeviCivita connection $\nabla^{*}$ of $S(T M)$ satisfy $\left(\nabla_{X_{\alpha}}^{*} X_{\beta}\right)(p)=0$ and $C\left(X_{0}, X_{\alpha}\right)=0$. Furthermore, we will assume that the 1-form $\tau$ vanishes on $S(T M)$. As an example we have the following.

Example 3.1 (Null cone of $\mathbb{R}_{1}^{n+2}$ ). Let $\mathbb{R}_{1}^{n+2}$ be the space $\mathbb{R}^{n+2}$ endowed with a semi-Euclidean metric $\bar{g}(x, y)=-x_{0} y_{0}+\sum_{a=0}^{n+1} x_{a} y_{a},\left(x=\sum_{A=0}^{n+1} x^{A} \partial x_{A}\right)$ where $\partial x_{A}:=\frac{\partial}{\partial x_{A}}$. Then, the null cone $\Lambda_{0}^{n+1}$ is given by the equation $x_{0}^{2}=\sum_{a=1}^{n+1} x_{a}^{2}, x_{0} \neq 0$. It is well-known (for example see the books $[7,8]$ ) that $\Lambda_{0}^{n+1}$ is a null hypersurface of $\mathbb{R}_{1}^{n+2}$, in which the radical distribution is spanned by a global vector field $E=\sum_{A=0}^{n+1} x_{A} \partial x_{A}$ on $\Lambda_{0}^{n+1}$. The transversal bundle is spanned by a global section $N$ given by $N=\frac{1}{2 x_{0}^{2}}\left\{-x_{0} \partial x_{0}+\right.$ $\left.\sum_{a=1}^{n+1} x_{a} \partial x_{a}\right\}$. Moreover, $E$ being the position vector field, one gets $\bar{\nabla}_{X} E=$ $\nabla_{X} E=X$, for any $X \in \Gamma(T M)$. Consequently, $A_{E}^{*} X+\tau(X) E+X=0$. Noticing that the operator $A_{E}^{*}$ is screen-valved, we infer from the last relation that $A_{E}^{*} X=-P X, \tau(X)=-\bar{g}(X, N)=-\lambda(X)$, for any $X \in \Gamma(T M)$. Next, any $X \in \Gamma\left(S\left(T \Lambda_{0}^{n+1}\right)\right)$ is expressed as $X=\sum_{a=1}^{n+1} \widetilde{X}_{a} \partial x_{a}$, where $\left\{\widetilde{X}_{1}, \ldots, \widetilde{X}_{n+1}\right\}$ satisfy $\sum_{a=1}^{n+1} x_{a} \widetilde{X}_{a}=0$. From the above calculations, we can clearly see that $\tau(X)=0$ for any $X \in \Gamma(S(T M))$.

The following result is important to this paper.

Theorem 3.2. [22] Let $(M, g)$ be a screen integrable null hypersurface of a Lorentzian manifold $(\bar{M}, \bar{g})$. Under null MCF, the squared norm $\left|A_{E}^{*}\right|_{s}^{2}$ of the screen shape operator $A_{E}^{*}$ evolves according to the following

$$
\frac{\partial\left|A_{E}^{*}\right|_{s}^{2}}{\partial t}=\Delta^{s}\left|A_{E}^{*}\right|_{s}^{2}+2 \operatorname{tr}^{s}\left(A_{E}^{*} \circ A_{N}\right)\left|A_{E}^{*}\right|_{s}^{2}-2\left|\nabla^{s} B\right|_{s}^{2},
$$

where $\left|A_{E}^{*}\right|_{s}^{2}=g^{\alpha \mu} g^{\gamma \beta} B_{\alpha \mu} B_{\gamma \beta}=\operatorname{tr}^{s}\left(A_{E}^{* 2}\right)$. 
Moreover, when $M$ is a space of constant curvature $c$ and each leaf $M^{\prime}$ of $S(T M)$ is Einstein, we can constract a family of metrics $g(t)$ appearing in the expression of $\left|A_{E}^{*}\right|_{s}^{2}$ above.

Example 3.3. Under null mean curvature flow, the induced metric $g$ evolves (see [22]) according to

$$
\frac{\partial g_{\alpha \beta}}{\partial t}=-2 \mathcal{S} C_{\alpha \beta}
$$

where $\mathcal{S}=\operatorname{tr}^{S}\left(A_{E}^{*}\right)$. From [7], the curvature tensors $R$ and $R^{*}$ of $M$ and $S(T M)$, respectively, are related by

$$
\begin{aligned}
g(R(X, Y) P Z, P W) & =g\left(R^{*}(X, Y) P Z, P W\right)+C(X, P Z) B(Y, P W) \\
& -C(Y, P Z) B(X, P W) .
\end{aligned}
$$

As $M$ is a space of constant curvature $c$, we have (see [7, p. 41])

$$
R(X, Y) P Z=c\{g(Y, P Z) X-g(X, P Z) Y\}, \forall X, Y, Z \in \Gamma(T M) .
$$

Considering (3.3) and (3.4), we derive

$$
-\mathcal{S} C_{\alpha \beta}=R i c_{\alpha \beta}^{*}(g)+c(n-1) g_{\alpha \beta}-g\left(A_{E}^{*} X_{\alpha}, A_{N} X_{\beta}\right),
$$

where $R i c^{*}$ denotes the Ricci tensor of $M^{\prime}$. Let $k_{\alpha}^{*}$ and $k_{\alpha}$ be the eigenvalues of $A_{E}^{*}$ and $A_{N}$, respectively, with respect to $\left\{X_{1}, \ldots, n\right\}$. Placing (3.5) in (3.2) we get

$$
\frac{\partial g_{\alpha \beta}}{\partial t}=2 \operatorname{Ric}_{\alpha \beta}^{*}(g)+2 \sigma g_{\alpha \beta},
$$

where $\sigma:=c(n-1)-k_{\alpha}^{*} k_{\beta}$. As $M^{\prime}$ is Einstein, we have $\operatorname{Ric}_{\alpha \beta}^{*}(g(0))=$ $\varpi g_{\alpha \beta}(0)$, where $\varpi$ is a constant. Set $g_{\alpha \beta}=k g_{\alpha \beta}(0)$, where $k$ is a positive constant. Then $\operatorname{Ric}_{\alpha \beta}^{*}(g)=\operatorname{Ric}_{\alpha \beta}^{*}(g(0))=\varpi g_{\alpha \beta}(0)=\frac{\varpi}{k} g_{\alpha \beta}$. Let $g_{\alpha \beta}(t)=$ $f(t) g_{\alpha \beta}(0)$ be a solution to (3.6), then

$$
\begin{aligned}
\frac{\partial g_{\alpha \beta}}{\partial t}=f^{\prime}(t) g_{\alpha \beta}(0) & =2 \operatorname{Ric}_{\alpha \beta}^{*}(f(t) g(0))+2 \sigma f(t) g_{\alpha \beta}(0) \\
& =2 \operatorname{Ric}_{\alpha \beta}^{*}(g(0))+2 \sigma f(t) g_{\alpha \beta}(0) \\
& =2 \varpi g_{\alpha \beta}(0)+2 \sigma f(t) g_{\alpha \beta}(0),
\end{aligned}
$$

in which we have used the fact that $M^{\prime}$ is an Einstein manifold. From (3.7) we have $f^{\prime}(t)=2 \sigma f(t)+2 \varpi$. Solving this ODE leads to $f(t)=$ $-\frac{\varpi}{\sigma}+\left(1+\frac{\varpi}{\sigma}\right) e^{2 \sigma t}$, and hence

$$
g_{\alpha \beta}(t)=\left(-\frac{\varpi}{\sigma}+\left(1+\frac{\varpi}{\sigma}\right) e^{2 \sigma t}\right) g_{\alpha \beta}(0), \quad \alpha, \beta \in\{1, \ldots, n\} .
$$

Observe that the flow will become singular at $t=\frac{1}{2 \sigma} \ln \left(\frac{\varpi}{\varpi+\sigma}\right)$. 
3.1. Existence and uniqueness of solutions to the PDE (3.1). In what follows, we use the local theory of PDE's, in [23], to study the existence and regularity of solutions to the semilinear parabolic PDE (3.1). Let us set

$$
X\left(\left|A_{E}^{*}\right|_{s}^{2}\right):=2 \operatorname{tr}^{s}\left(A_{E}^{*} \circ A_{N}\right)\left|A_{E}^{*}\right|_{s}^{2}-2\left|\nabla^{s} B\right|_{s}^{2}
$$

We further suppose that $X$ is $C^{\infty}$ in its arguments. Substituting (3.8) back in (3.1), one gets the following initial value PDE.

$$
\frac{\partial\left|A_{E}^{*}\right|_{s}^{2}}{\partial t}=\Delta^{s}\left|A_{E}^{*}\right|_{s}^{2}+X\left(\left|A_{E}^{*}\right|_{s}^{2}\right), \quad\left|A_{E}^{*}\right|_{s}^{2}(0)=f .
$$

By integrating (3.9) we get the following

$$
\left|A_{E}^{*}\right|_{s}^{2}(t)=e^{t \Delta^{s}} f+\int_{0}^{t} e^{(t-q) \Delta^{s}} X\left(\left|A_{E}^{*}\right|_{s}^{2}(q)\right) d q=\Phi\left|A_{E}^{*}\right|_{s}^{2}(t) .
$$

Next, we setup a Banach space $\mathcal{B}\left([0, T], C^{1}(M)\right)$ preserved by the map $\Phi$ and hence show that (3.10) has a solution, using the contraction mapping principle (see [23] for more details). We suppose further that $f \in C^{1}(M)$, which is a Banach space of continuously differentiable functions on $M$. Let $C(M)$ be another Banach space, then the following conditions holds [23].

(1) $e^{t \Delta^{s}}: C^{1}(M) \longrightarrow C^{1}(M)$ is a strongly continuous semigroup, for $t \geq 0$

(2) $X: C^{1}(M) \longrightarrow C(M)$ is a locally Lipschitz map,

(3) $e^{t \Delta^{s}}: C(M) \longrightarrow C^{1}(M)$, for $t>0$, and for some $\gamma<1$,

(4) $\left\|e^{t \Delta^{s}}\right\|_{\mathcal{L}\left(C, C^{1}\right)} \leq C t^{-\gamma}$, for $t \in(0,1]$.

Now, using the above conditions we have the following.

Theorem 3.4. Let $(M, g)$ be a compact null hypersurface of $(\bar{M}, \bar{g})$. Under conditions (1)-(4) the $\operatorname{PDE}(3.9)$, with initial data $f \in C^{1}(M)$, has a unique solution $\left|A_{E}^{*}\right|_{s}^{2} \in \mathcal{B}\left([0, T], C^{1}(M)\right)$ where $T>0$ is estimable from below in terms of $\|f\|_{C^{1}}$.

Proof. From the above conditions it is easy to see that $\Phi$ acts on $\mathcal{B}\left([0, T], C^{1}(M)\right)$, for each $T>0$. Fix $\epsilon>0$, and set

$$
\mathcal{G}=\left\{\left|A_{E}^{*}\right|_{s}^{2} \in \mathcal{B}\left([0, T], C^{1}(M)\right),\left|A_{E}^{*}\right|_{s}^{2}(0)=f,\left\|\left|A_{E}^{*}\right|_{s}^{2}(t)-f\right\|_{C^{1}} \leq \epsilon\right\} .
$$

Consequently, we want to pick $T$ small enough such that $\Phi: \mathcal{G} \longrightarrow \mathcal{G}$ is a contraction. By (1) we can choose $T_{1}$ so that $\left\|e^{t \Delta^{s}} f-f\right\|_{C^{1}} \leq \frac{\epsilon}{2}$, for $t \in\left[0, T_{1}\right]$. Next, if $\left|A_{E}^{*}\right|_{s}^{2} \in \mathcal{G}$, by (2) we have $\left\|X\left(\left|A_{E}^{*}\right|_{s}^{2}(q)\right)\right\|_{C} \leq K_{1}$, for $q \in\left[0, T_{1}\right]$. Then by (4) we have $\left\|\int_{0}^{t} e^{(t-q) \Delta^{s}} X\left(\left|A_{E}^{*}\right|_{s}^{2}(q)\right) d q\right\|_{C^{1}} \leq C_{\gamma} t^{1-\gamma} K_{1}$. If we pick $T_{2} \leq T_{1}$ small enough, this will be $\leq \frac{\epsilon}{2}$ for $t \in\left[0, T_{2}\right]$, hence 
$\Phi: \mathcal{G} \longrightarrow \mathcal{G}$ provided $T \leq T_{2}$. To arrange that $\Phi$ is a contraction we use (2) to obtain

$$
\left\|X\left(\left|A_{E}^{*}\right|_{s}^{2}(q)\right)-X\left(\mid \widetilde{\left.A_{E}^{*}\right|_{s} ^{2}}(q)\right)\right\|_{C} \leq K\left\|\left|A_{E}^{*}\right|_{s}^{2}(q)-{\widetilde{A_{E}^{*}}}_{\mid}^{2}(q)\right\|_{C^{1}},
$$

for all $\left|A_{E}^{*}\right|_{s}^{2}, \mid \widetilde{\left.A_{E}^{*}\right|_{s} ^{2}} \in \mathcal{G}$. Hence, for $t \in\left[0, T_{2}\right]$,

$$
\begin{aligned}
\| \Phi\left(\left|A_{E}^{*}\right|_{s}^{2}\right)(t) & -\Phi\left(\mid \widetilde{\left.A_{E}^{*}\right|_{s} ^{2}}\right)(t) \|_{C^{1}} \\
& =\left\|\int_{0}^{t} e^{t \Delta^{s}}\left(X\left(\left|A_{E}^{*}\right|_{s}^{2}(q)\right)-X\left(\widetilde{\left|A_{E}^{*}\right|_{s}^{2}}(q)\right)\right) d q\right\|_{C^{1}} \\
& \leq C_{\gamma} t^{1-\gamma} K \sup \left\|\left|A_{E}^{*}\right|_{s}^{2}(q)-\left|\widetilde{A_{E}^{*}}\right|_{s}^{2}(q)\right\|_{C^{1}},
\end{aligned}
$$

from which if $T \leq T_{2}$ is chosen small enough, we have $C_{\gamma} t^{1-\gamma} K<1$, which makes $\Phi$ a contraction map on $\mathcal{G}$. Therefore, $\Phi$ has a unique fixed point $\left|A_{E}^{*}\right|_{s}^{2} \in \mathcal{G}$ solving the PDE (3.9), which completes the proof.

3.2. Singularities from the PDE (3.1). Here, we discuss some singularities associated to (3.1) in the case $M$ is a screen conformal null hypersurface. A null hypersurface $(M, g)$ of a semi-Riemannian manifold $(\bar{M}, \bar{g})$ is called screen conformal $[7$, p. 51] if there exist a non-vanishing smooth function $\psi$ on a neighborhood $\mathcal{U}$ in $M$ such that $A_{N}=\psi A_{E}^{*}$, or equivalently,

$$
C(X, P Y)=\psi B(X, Y), \quad \forall X, Y \in \Gamma(T M) .
$$

We say that $M$ is screen homothetic if $\psi$ is a constant function on $M$.

Example 3.5. Consider the null cone in Example 3.1. By straightforward calculation, one gets $\bar{g}\left(\nabla_{E} X, E\right)=-\sum_{a=1}^{n+1} x_{a} X_{a}=0$, which implies that $\nabla_{E} X \in \Gamma\left(S\left(T \Lambda_{0}^{n+1}\right)\right)$. Hence, $A_{N} E=0$. Using Gauss-Codazzi equations, we calculate $C(X, Y)=\bar{g}\left(\nabla_{X} Y, N\right)=\bar{g}\left(\bar{\nabla}_{X} Y, N\right)=-\frac{1}{2 x_{0}^{2}} g(X, Y)$, for any $X, Y \in \Gamma\left(S\left(T \Lambda_{0}^{n+1}\right)\right)$. Consequently, $A_{N} X=-\frac{1}{2 x_{0}^{2}} P X$, for any $X, Y \in \Gamma\left(S\left(T \Lambda_{0}^{n+1}\right)\right)$. Thus, deduce that $A_{N} X=\frac{1}{2 x_{0}^{2}} A_{E}^{*} X$, for any $X \in$ $\Gamma\left(S\left(T \Lambda_{0}^{n+1}\right)\right)$. Hence, $\Lambda_{0}^{n+1}$ is screen globally conformal null hypersurface of $\mathbb{R}_{1}^{n+2}$, with a positive conformal factor $\psi=\frac{1}{2 x_{0}^{2}}$ globally define on $\Lambda_{0}^{n+1}$.

From the result of Theorem 3.2, we notice that one gets a quadratic term in $\left|A_{E}^{*}\right|_{s}^{2}$ when $M$ is screen conformal. More precisely, when $M$ is screen conformal, we have $\operatorname{tr}^{s}\left(A_{E}^{*} \circ A_{N}\right)=\psi \operatorname{tr}^{s}\left(A_{E}^{* 2}\right)=\psi\left|A_{E}^{*}\right|_{s}^{2}$. Thus, from (3.1) we have

$$
\frac{\partial\left|A_{E}^{*}\right|_{s}^{2}}{\partial t}=\Delta^{s}\left|A_{E}^{*}\right|_{s}^{2}+2 \psi\left|A_{E}^{*}\right|_{s}^{4}-2\left|\nabla^{s} B\right|_{s}^{2}
$$


Relation (3.12) indicates that there will also be singularities in null MCF if the initial null hypersurface $M_{0}$ is screen conformal, as the quantity $\left|A_{E}^{*}\right|_{s}^{2}$ blows-up in finite time. In fact, the above equation resembles the PDE; $u_{t}=\Delta u+u^{2}$, which has solutions which blows up at infinity. When this happens we say that $t:=T$ is singular time for the null MCF. Moreover, we have the following.

Theorem 3.6. [22] Let $M_{0}$ be a mean convex, screen conformal null hypersurface. If $\left|A_{E}^{*}\right|_{s}^{2}$ is not bounded as $t \longrightarrow T<+\infty$ during the null MCF of a compact null hypersurface, then it must satisfy the following lower bound for its blow-up rate:

$$
\max _{p \in M}\left|A_{E}^{*}\right|_{s}^{2}(p, t) \geq \frac{1}{2 \psi(T-t)},
$$

for every $t \in[0, T)$. Hence, $\lim _{t \longrightarrow T} \max _{p \in M}\left|A_{E}^{*}\right|_{s}^{2}(p, t)=+\infty$.

In accordance to Theorem 3.6, let $T$ be the maximal time of existance of null mean curvature flow, such that $\psi>0$. If there exists a constant $\wp>1$ such that we have the upper bound

$$
\max _{p \in M}\left|A_{E}^{*}\right|_{s}(p, t) \leq \frac{\wp}{\sqrt{2 \psi(T-t)}},
$$

we say that the flow is developing a type I singularity at $T$. In the event that such a constant does not exist, that is $\lim \sup _{t \rightarrow T} \max _{p \in M}\left|A_{E}^{*}\right|_{s}(p, t) \sqrt{T-t}=$ $+\infty$, we say that we have a type II singularity.

One of the major tools in studying the singularities associated to mean curvature flow of Riemannan surfaces is Huisken's monotonicity formula [13]. It is based on a Gaussian backward heat kernel and the first variation formula for Riemannian surfaces. Due to the inter-relationships between some geometric quantities on null hypersurfaces (and submanifolds in general) and the fact that the normal bundle, $T M^{\perp}$, is a subbundle of the tangent bundle, $T M$, of the null hypersurface $M$, such monotonicity formula cannot be used to investigate singularities in our case. Thus, we need a null version of it. To state and prove a monotonicity formula for null hypersurfaces moving by their mean curvature, we need a null variation formula. To do this, let $U$ be a vector field on $M$, whose screen component $P U$ has a compact support in the interior of $M_{t}$. Then, according to (2.1), $U$ has a unique decomposition $U=P U+\bar{g}(U, N) E$. Using this decomposition of $U$, we have the following. 
Proposition 3.7. Let $(M, g)$ be a compact screen conformal null hypersurface of $(\bar{M}, \bar{g})$. Let $\mathcal{U}$ be a neighborhood of $M$ and let $U$ be a compactly supported vector field on the screen distribution of $M$. If $\mathcal{U}_{t}$ is a 1-parameter family of null hypersurfaces tangent at $t=0$ to the variation $U$, then

$$
\int_{\mathcal{U}} \operatorname{div}^{S(T M)}(U) d \mu=-\int_{\mathcal{U}} \bar{g}(H, U) d \mu,
$$

where $d \mu$ denotes the volume form with respect to $g$ restricted to $S(T M)$ and $\operatorname{div}^{S(T M)}(\cdot)$ is the divergence operator with respect to $S(T M)$.

Proof. Let $(M, g)$ be a null hypersurface, and let $\mathcal{U}$ be a neighborhood of $M$. Let $f: \mathcal{U} \times(-\varepsilon, \varepsilon) \rightarrow M$ be a one-parameter variation supported in the interior of $\mathcal{U}$. Let $t$ denote the coordinate on $(-\varepsilon, \varepsilon)$, and let $U:=f_{*}\left(\partial_{t}\right)$. In this regard, $\mathcal{U}:=f(\mathcal{U})$ evolves to $\mathcal{U}_{t}:=f(\mathcal{U}, t)$. Flow $U$ determines an endomorphism field $\mathcal{A}_{U}$ along $f$. This endomorphism decomposes into a skew-symmetric part, which rotates $S(T \mathcal{U})$ but preserves volume (here volume is taken with respect to degenerate metric $g$ restricted to $S(T M)$ ), and a symmetric part. The derivative at $t=0$ of the area of an infinitesimal plane tangent to $S\left(T \mathcal{U}_{t}\right)$ is then the negative of the trace of $\mathcal{A}_{U}$ restricted to $S(T \mathcal{U})$. In fact, let $d \mu$ denote the induced volume form on $\mathcal{U}_{t}$, then

$$
\operatorname{vol}\left(\mathcal{U}_{t}\right)=\int_{\mathcal{U}} f_{t}^{*} d \mu
$$

where $f_{t}^{*} d \mu$ denotes the pullback of the volume form $d \mu$ on $\mathcal{U}_{t}$ under $f_{t}$. But $f_{t}^{*} d \mu\left(X_{1}, \ldots, X_{n}\right)=\left.d \mu\right|_{f_{t}}\left(d f_{t} X_{1}, \ldots, d f_{t} X_{n}\right)=\left.\operatorname{det}\left(d f_{t}\right) d \mu\left(X_{1}, \ldots, X_{n}\right)\right|_{f_{t}}=$ $\operatorname{det}\left(d f_{t}\right)$, where $\left\{X_{1}, \ldots, X_{n}\right\}$ is an orthonormal frame field along $\mathcal{U}$. Let $g_{\alpha \beta}^{t}=\bar{g}\left(d f_{t} X_{\alpha}, d f_{t} X_{\beta}\right)$ and $g(t)=\operatorname{det}\left(g_{\alpha \beta}^{t}\right)$, then $\operatorname{det}\left(d f_{t}\right)=\sqrt{g(t)}$. Thus, from (3.14), we have

$$
\left.\frac{d}{d t}\right|_{t=0} \operatorname{vol}\left(\mathcal{U}_{t}\right)=\left.\int_{\mathcal{U}} \frac{d}{d t}\right|_{t=0} \operatorname{det}\left(d f_{t}\right) d \mu_{0}=\left.\int_{\mathcal{U}} \frac{1}{2} \frac{d g}{d t}\right|_{t=0} d \mu .
$$

By simple calculations, we have

$$
\left.\frac{1}{2} \frac{\partial}{\partial t} g_{\alpha \alpha}^{t}\right|_{t=0}=\left.g\left(\nabla_{U} X_{\alpha}^{t}, X_{\alpha}^{t}\right)\right|_{t=0}=\left.g\left(\nabla_{X_{\alpha}^{t}} U, X_{\alpha}^{t}\right)\right|_{t=0},
$$

where $X_{\alpha}^{t}=d f \cdot X_{\alpha}$. Hence, considering (3.15) and (3.16), we get

$$
\left.\frac{d}{d t}\right|_{t=0} \operatorname{vol}\left(\mathcal{U}_{t}\right)=\int_{\mathcal{U}} \operatorname{div}^{S(T M)}(U) d \mu
$$


From (3.17) and the decomposition of $U$ we have

$$
\left.\frac{d}{d t}\right|_{t=0} \operatorname{vol}\left(\mathcal{U}_{t}\right)=\int_{\mathcal{U}} \operatorname{div}^{S(T M)}(P U) d \mu+\int_{\mathcal{U}} \operatorname{div}^{S(T M)}(\bar{g}(U, N) E) d \mu .
$$

As $P U$ is compactly supported on $S(T \mathcal{U})$, Stoke's theorem reduces (3.18) to

$$
\left.\frac{d}{d t}\right|_{t=0} \operatorname{vol}\left(\mathcal{U}_{t}\right)=\int_{\mathcal{U}} \operatorname{div}^{S(T M)}(\bar{g}(U, N) E) d \mu
$$

By simple calculations, while considering (2.6), we have

$$
\begin{aligned}
g\left(\nabla_{X_{\alpha}} \bar{g}(U, N) E, X_{\alpha}\right) & =g\left(X_{\alpha}, E\right) X_{\alpha} \cdot \bar{g}(U, N)+\bar{g}(U, N) g\left(\nabla_{X_{\alpha}} E, X_{\alpha}\right) \\
& =-B\left(X_{\alpha}, X_{\alpha}\right) \bar{g}(U, N), \quad \forall \alpha \in\{1, \ldots, n\} .
\end{aligned}
$$

Plugging (3.20) in (3.19) we get

$$
\left.\frac{d}{d t}\right|_{t=0} \operatorname{vol}\left(\mathcal{U}_{t}\right)=-\int_{\mathcal{U}} \sum_{\alpha=1}^{n} B\left(X_{\alpha}, X_{\alpha}\right) \bar{g}(U, N) d \mu,
$$

which concludes the proof.

Proposition 3.7 gives the null first variational formula. Now, we are in position to state and prove a general monotonicity formula for null hypersurfaces evolving by their mean curvature. To this end, let us first define a backwards heat kernel (see details in [13]) for such evolution. Let us define a backward heat kernel $\rho$ at $\left(0, t_{0}\right)$ by

$$
\rho(X, t):=\frac{1}{\left(4 \pi\left(t_{0}-t\right)\right)^{\frac{n}{2}}} \exp \left(-\frac{|X|^{2}}{4 \pi\left(t_{0}-t\right)}\right), \quad t<t_{0},
$$

where $|\cdot|^{2}:=\widehat{g}(\cdot, \cdot)$. Notice that $(3.22)$ is a Gaussian function. This function is frequently used as a backward heat kernel due to its nice properties (see for instance [13]). With such a heat kernel, we have the following null monotocity formula.

Theorem 3.8. Let $M_{t}$ be a screen conformal null hypersurface evolving by mean curvature and satisfying Definition 2.1. Then

$$
\frac{d}{d t} \int_{\mathcal{U}_{t}} \rho(X, t) d \mu=-\int_{\mathcal{U}_{t}} \rho(X, t)\left\{\psi \mathcal{S}^{2}+\mathcal{S} \frac{\lambda(X)}{\left(t_{0}-t\right)}+\frac{\lambda(X)^{2}}{4\left(t_{0}-t\right)^{2}}\right\} d \mu,
$$

where $\lambda(X)=\bar{g}(X, N)$. 
Proof. By a straightforward calculation while considering the evolution equation $\frac{d}{d t} \mu=-\bar{g}(H, \widehat{H}) d \mu$, where $\widehat{H}=\sum_{a=0}^{n} C\left(X_{a}, P X_{a}\right) E$ and the fact $M$ is screen conformal we derive

$$
\begin{aligned}
\frac{d}{d t} & \int_{\mathcal{U}_{t}} \rho(X, t) d \mu \\
& =\int_{\mathcal{U}_{t}}\left\{\frac{\partial \rho(X, t)}{\partial t}+\bar{g}(\nabla \rho(X, t), H)\right\} d \mu+\int_{\mathcal{U}_{t}} \rho(X, t) \frac{\partial d \mu}{\partial t} \\
& =\int_{\mathcal{U}_{t}}\left\{\frac{\partial \rho(X, t)}{\partial t}+\bar{g}(\nabla \rho(X, t), H)\right\} d \mu-\int_{\mathcal{U}_{t}} \rho(X, t) \bar{g}(\widehat{H}, H) d \mu \\
& =\int_{\mathcal{U}_{t}}\left\{\frac{\partial \rho(X, t)}{\partial t}+\bar{g}(\nabla \rho(X, t), H)\right\} d \mu-\int_{\mathcal{U}_{t}} \psi \rho(X, t) \mathcal{S}^{2} d \mu,
\end{aligned}
$$

where $\nabla f$ denotes the gradient of a smooth function $f$ with respect to $\widehat{g}$. From (3.22) we have

$$
\frac{\partial \rho(X, t)}{\partial t}=\frac{n}{2\left(t_{0}-t\right)} \rho(X, t)-\frac{|X|^{2}}{4\left(t_{0}-t\right)^{2}} \rho(X, t),
$$

and $\nabla \rho(X, t)=-\frac{1}{2\left(t_{0}-t\right)} \rho(X, t) X$.

Putting (3.24) and (3.25) in (3.23) we have

$$
\begin{aligned}
\frac{d}{d t} \int_{\mathcal{U}_{t}} \rho(X, t) d \mu & =\int_{\mathcal{U}_{t}}\left\{\frac{n}{2\left(t_{0}-t\right)} \rho(X, t)-\frac{|X|^{2}}{4\left(t_{0}-t\right)^{2}} \rho(X, t)\right. \\
& \left.-\frac{\rho(X, t)}{2\left(t_{0}-t\right)} \bar{g}(X, H)\right\} d \mu-\int_{\mathcal{U}_{t}} \psi \rho(X, t) \mathcal{S}^{2} d \mu \\
& =-\int_{\mathcal{U}_{t}} \rho(X, t)\left\{\psi \mathcal{S}^{2}+\frac{1}{\left(t_{0}-t\right)} \bar{g}(X, H)+\frac{|X|^{2}}{4\left(t_{0}-t\right)^{2}}\right\} d \mu \\
& +\int_{\mathcal{U}_{t}} \frac{\rho(X, t)}{2\left(t_{0}-t\right)} \bar{g}(X, H) d \mu+\int_{\mathcal{U}_{t}} \frac{n}{2\left(t_{0}-t\right)} \rho(X, t) d \mu .
\end{aligned}
$$

Now, using the first variation formula in Proposition 3.7 with $U=\frac{\Phi}{2\left(t_{0}-t\right)} X$, we derive

$$
\int_{\mathcal{U}_{t}} \frac{\rho(X, t)}{2\left(t_{0}-t\right)} \bar{g}(X, H) d \mu=-\int_{\mathcal{U}_{t}} \rho(X, t)\left\{\frac{n}{2\left(t_{0}-t\right)}-\frac{|P X|^{2}}{4\left(t_{0}-t\right)^{2}}\right\} d \mu .
$$


Finally, plugging (3.27) in (3.26) and using $X=P X+\bar{g}(X, N) E$ we get

$$
\begin{aligned}
\frac{d}{d t} \int_{\mathcal{U}_{t}} \rho(X, t) d \mu & =-\int_{\mathcal{U}_{t}} \rho(X, t)\left\{\psi \mathcal{S}^{2}+\frac{1}{\left(t_{0}-t\right)} \bar{g}(X, H)+\frac{|X|^{2}}{4\left(t_{0}-t\right)^{2}}\right\} d \mu \\
& +\int_{\mathcal{U}_{t}} \frac{|P X|^{2}}{4\left(t_{0}-t\right)^{2}} \rho(X, t) d \mu \\
& =-\int_{\mathcal{U}_{t}} \rho(X, t)\left\{\psi \mathcal{S}^{2}+\frac{\bar{g}(X, H)}{\left(t_{0}-t\right)}+\frac{\bar{g}(X, N)^{2}}{4\left(t_{0}-t\right)^{2}}\right\} d \mu,
\end{aligned}
$$

which proves our result and the proof is complete.

Theorem 3.8 gives the null monotonicity formula for a screen conformal lightlike hypersurface evolving by it mean curvature.

Let $(0, T)$ be a Type I singularity and consider the rescaled mean curvature flow

$$
\widetilde{F}(x, \widetilde{s})=\frac{F(x, t)}{\sqrt{2 \psi(T-t)}}, \quad \widetilde{s}(t)=-\frac{1}{2 \psi} \log (T-t) .
$$

Then

$$
\frac{d \widetilde{F}(x, \widetilde{s})}{d \widetilde{s}}=\widetilde{\mathbf{H}}(x, \widetilde{s})+\widetilde{F}(x, \widetilde{s}),
$$

on $M \times[0,+\infty)$. Using the above rescaling and Theorem 3.8 , we have the following important result.

Corollary 3.9. If the surface $\widetilde{M}_{\widetilde{s}}$ satisfies the rescaled equation (3.28), then

$$
\frac{d}{d \widetilde{s}} \int_{\widetilde{F}_{\widetilde{s}}} \rho(\widetilde{F}, \widetilde{s}) d \mu=-\int_{\widetilde{F}_{\widetilde{s}}} \rho(\widetilde{F}, \widetilde{s})\left\{\psi \mathcal{S}^{2}+2 \mathcal{S} \lambda(\widetilde{F})+\lambda(\widetilde{F})^{2}\right\} d \widetilde{\mu}_{\widetilde{s}}
$$

where $\widetilde{F}_{\widetilde{s}}=\widetilde{F}_{\widetilde{s}}(M), \rho(\widetilde{F})=\exp \left(-\frac{1}{2}|\widetilde{F}|^{2}\right)$ and $\lambda(\widetilde{F})=\bar{g}(\widetilde{F}, N)$.

Next, we use Corollary 3.9 to study the behavior of $\widetilde{M}_{\widetilde{s}}$ as $\widetilde{s} \longrightarrow \infty$.

Proposition 3.10. Let $[0, T)$ be a type I singularity such that (3.13) holds. Then there is $x \in M$ such that $\widetilde{F}(x, \widetilde{s})$ as defined in (3.28) remains bounded for $\widetilde{s} \longrightarrow \infty$.

Proof. In view of the null mean curvature flow relation of Definition 2.1, it is easy to show that $|F(x, t)| \leq \mathcal{C}_{0}(M)$, where $\mathcal{C}_{0}(M)$ is a non-zero constant on $M$ independent of time, which ends the proof.

Notice that $F_{t} \cap \mathbb{B}_{\mathcal{C}(M)}(0)$ can not be empty for $\mathcal{C}(M)$ large enough, otherwise the time $T$ can not be the first time singularity. Therefore, $\widetilde{F}(\cdot, \widetilde{s})$ is indeed locally uniformly bounded. 
Theorem 3.11. Let $[0, T)$ be a type I singularity such that (3.13) holds. Then for each sequence $\widetilde{s}_{j} \longrightarrow \infty$ there is a subsequence $\widetilde{s}_{j k}$ such that $\widetilde{M}_{\widetilde{s}_{j k}}$ converges smoothly to an immersed nonempty limiting surface $\widetilde{M}_{\infty}$.

Proof. A proof uses similar arguments as that in [13].

Now, using the rescaled null monotonicity formula in Corollary 3.9 we have the following result about the limiting surface $\widetilde{M}_{\infty}$.

Theorem 3.12. For $\psi \leq 1$, each limiting surface $\widetilde{M}_{\infty}$ as per Theorem 3.11 satisfies

$$
\mathcal{S}=\bar{g}(\mathbf{X}, N), \quad \mathbf{X}:=-\left(\frac{\psi}{1+\sqrt{1-\psi}}\right)^{-1} X,
$$

where $X$ is the position vector and $\mathcal{S}$ is the null mean curvature and $N$ is the lightlike transversal vector, such that $\mathbf{H}=\mathcal{S} N$ is the mean curvature vector.

Proof. From the lightlike monotonicity formula of Corollary 3.9 and the fact that the integral of $\rho$ is positve, we have

$$
\int_{\widetilde{s}_{0}}^{\infty} \int_{\widetilde{F}_{\widetilde{s}}} \rho(\widetilde{F}, \widetilde{s})\left\{\psi \mathcal{S}^{2}+2 \mathcal{S} \lambda(\widetilde{F})+\lambda(\widetilde{F})^{2}\right\} d \widetilde{\mu}_{\widetilde{s}} d \widetilde{s}<\infty
$$

and the result follows immediately in view of the uniform estimates in Proposition 2.3 as in [13].

Observe that the relation (3.30) in Theorem 3.12 is a second order elliptic equation. For any solution $F_{0}$ to $(3.30)$, there is an associated self-similar solution of the null mean curvature flow, given by $F(, t)=(2(T-t))^{1 / 2} F_{0}$. Thus, from the above we have the following.

Theorem 3.13. The null mean curvature flow $F=\left\{F_{t}\right\}$ at a type I singularity $\left(x_{0}, T\right)$ is asymptotically self-similar, in the sense that $[2(T-t)]^{-1 / 2}\left(F_{t}-x_{0}\right)$ converges locally uniformly to a solution $F$ of (3.30).

In Theorem 3.13, we obtained a self-similar blow-up solution at type I singularity, which is an ancient solution, namely it exists from time $t=-\infty$. At type II singularity, there is a blow-up solution which is an eternal solution. To see this, we choose the blow-up points $\left(x_{k}, t_{k}\right)$ as follows. For each integer $k \geq 1$, let $t_{k} \in\left[0, T-\frac{1}{k}\right]$ and $x_{k} \in F_{t_{k}}$, such that

$$
\left|A_{E}^{*}\right|_{s}^{2}\left(x_{k}, t_{k}\right)\left(T-\frac{1}{k}-t_{k}\right)=\max _{t \leq T-\frac{1}{k}, x \in F_{t}}\left|A_{E}^{*}\right|_{s}^{2}(x, t)\left(T-\frac{1}{k}-t\right) .
$$


Let us make the dilation

$$
F^{k}(y, \widetilde{s})=\epsilon_{k}^{-1}\left[F\left(x_{k}+\epsilon_{k} y, t_{k}+\epsilon_{k}^{2} \widetilde{s}\right)-F\left(x_{k}, t_{k}\right)\right],
$$

where $\epsilon_{k}=\left(\left.\left|A_{E}^{*}\right|\right|_{s}\left(x_{k}, t_{k}\right)\right)^{-1}$. Since the singularity is of Type II, the right hand side of (3.32) tends to $+\infty$ as $k \longrightarrow+\infty$. Hence, for any finished $\widetilde{s} \in$ $\left[-\epsilon_{k}^{-2} t_{k}, \epsilon_{k}^{-2}\left(T-\frac{1}{k}-t\right)\right]$, the mean curvature of the rescaled hypersurface $F^{k}$ satisfies

$$
\left|A_{E}^{*}\right|_{s}^{2} \leq \frac{T-\frac{1}{k}-t_{k}}{T-\frac{i}{k}-t}=\frac{T-\frac{1}{k}-t_{k}}{T-\frac{i}{k}-t_{k}-\epsilon_{k}^{2} \widetilde{s}} \longrightarrow 1 \text {, as } k \longrightarrow+\infty .
$$

It follows that for any $\widetilde{s}^{*}>1$ and any $\epsilon>0$ there exists $k_{0}$ such that

$$
\max _{F^{k}}\left|A_{E}^{*}\right|_{s}(\cdot, \widetilde{s}) \leq 1+\epsilon,
$$

for any $k \geq k_{0}$ and $\widetilde{s} \in\left[-\widetilde{s}^{*}, \widetilde{s}^{*}\right]$. By similar arguments as in [21], the second fundamental form is uniformly bounded and all derivatives of the second fundamental forms of $F^{k}(\cdot, \widetilde{s})$ are uniformly bounded. Hence, by the Arzela-Ascoli theorem, we can extract a subsequence of $F^{k}(\cdot, \widetilde{s})$ which converges uniformly on any compact subsets of $\mathbb{R}^{n+2} \times \mathbb{R}^{1}$ to a blow-up solution $F^{\infty}(\cdot, \widetilde{s})$ (see [6], [16], [20] for more details).

\section{Hanarck estimates}

In classical Riemannian (or semi-Riemannian) geometry, a submanifold $M$ in a flat space $\bar{M}$ is called a translating soliton [10] if there exists a constant vector $\bar{K}$ in $\bar{M}$ such that $\bar{K}=K+\mathbf{H}$ on $\bar{M}$, where $K$ is its tangential component and $\mathbf{H}$ is the mean curvature vector of $M$ in $\bar{M}$. Equivalently, $M$ is a translating soliton if $\mathbf{H}=\bar{K}^{\perp}$, where $\bar{K}^{\perp}$ is the projection of $\bar{K}$ in $\bar{M}$ on the normal bundle of $M$. Consequently, the 1-parameter family of submanifolds $M_{t}$ defined by $M_{t}=M+t \bar{K}$ for $t \in \mathbb{R}$ is a solution to the classical MCF (see [9], [14], [15] and many more references therein). Soliton solutions have proved fundamentally important in mean curvature flow studies, for instance singularities are locally modeled on soliton solutions (see [21] and many more references therein). In [12], Hamiliton used translating solitons to prove some harnack estimates for MCF of Riemannian hypersurfaces.

We have seen, in the previous section, that the evolution equations for null MCF possesses some analogies with those in classical MCF of Riemannian hypersurfaces (see some of such evolution equations in [13], [14], [15] and many more references therein). Thus, singularities are bound to occur as in the usual MCF. Furthermore, translators or translating solitons can equally 
be defined in null MCF to facilitate the study of such singularities as well as in the establishment of null Harnack inequalities which is the main aim of this section. Note, however, that in null MCF we can not use the above definition of translating solitons due to the fact that the normal bundle of a null hypersurface is a subbundle of its tangent bundle. Furthermore, in null MCF the null hypersurface under consideration is evolving in the transversal direction. From the decomposition of $T \bar{M}$ in (2.2), any vector $\bar{K}$ of $\bar{M}$ can be uniquely writen as $\bar{K}=K+K^{t r}$, where $K$ and $K^{t r}$ are its tangential and tranversal components, respectively. Thus, for null MCF, we have the following definition.

Definition 4.1. A solution $M_{t}$ of null MCF will be called a null translating soliton if its null mean curvature vector $\mathbf{H}$ satisfy $\mathbf{H}=\bar{K}^{t r}$.

As $\mathbf{H}=\mathcal{S} N$, we immediately get $\mathcal{S}=\bar{g}\left(K^{t r}, E\right)$, where $E$ spans the radical distribution of $M_{t}$. Suppose a solution $M_{t}$ to the null MCF in Definition 2.1 translates in the direction of the constant vector $\bar{K}=\left(\bar{K}^{0}, \ldots, \bar{K}^{n+1}\right)$, for $i \in\{0, \ldots, n+1\}$, and let $K=\left(K^{0}, \ldots, K^{n+1}\right)$ be its tangential component. Then the transversal component of $\bar{K}$ must be $\mathcal{S} N^{i}$ to solve the null MCF. Locally, the tangential component $K$ can be writen as $K^{i}=$ $K^{a} \frac{\partial y^{i}}{\partial x_{a}}:=K^{a} \nabla_{X_{a}} y^{i}$, where $y^{i}=F^{i}\left(x_{a}, t\right)$. Also, with respect to the nondegenerate metric $\widehat{g}$ its covector is $K_{a}=\widehat{g}_{a b} K^{b}$, for any $a, b \in\{0, \ldots, n\}$. Then differentiating $\bar{K}^{i}=K^{i}+\mathcal{S} N^{i}$ and using the facts $\nabla_{X_{a}} \bar{K}^{i}=0(\bar{K}$ is a constant vector), (2.4) and $\tau=0$ we get

$$
g^{[b c]}\left(\nabla_{X_{a}} K_{b}-\mathcal{S} C_{a b}\right) \nabla_{X_{c}} y^{i}+\left(\nabla_{X_{a}} \mathcal{S}+g^{[b c]} B_{a b} K_{c}\right) N^{i}=0 .
$$

Taking the $\bar{g}$-product of (4.1) with $N^{i}$ and observing that $\bar{g}\left(\nabla_{X_{c}} y^{i}, N^{i}\right) \neq 0$, we get the two relations

$$
\nabla_{X_{a}} K_{b}=\mathcal{S} C_{a b} \text { and } \nabla_{X_{a}} \mathcal{S}+B_{a b} K_{b}=0 .
$$

By simple calculations while considering the first relation of (4.2), we have

$$
\left(\mathcal{L}_{K} g\right)\left(X_{\alpha}, X_{\beta}\right)=\nabla_{X_{\alpha}} K_{\beta}+\nabla_{X_{\beta}} K_{\alpha}=\mathcal{S}\left(C_{\alpha \beta}+C_{\beta \alpha}\right) .
$$

Hence, from the evolution equation of $g$ (see [22]), if the local second fundamental form $C$ is symmetric, the degenerate metric $g$ evolves by the Lie derivative with respect to $K$, that is;

$$
\frac{\partial}{\partial t} g_{\alpha \beta}=-\left(\mathcal{L}_{K} g\right)_{\alpha \beta}, \quad \alpha, \beta \in\{1, \ldots, n\} .
$$


The vector field $\bar{K}$ is said to be conformal [7, p. 259] if

$$
\left(\mathcal{L}_{\bar{K}} \bar{g}\right)(\bar{X}, \bar{Y})=2 \Psi \bar{g}(\bar{X}, \bar{Y}), \quad \forall \bar{X}, \bar{Y} \in \Gamma(T \bar{M}),
$$

where $\Psi$ is a scalar function on $\bar{M}$. In particular, $\bar{K}$ is homothetic or Killing vector field according as $\Psi$ is non-zero constant or zero respectively. $\bar{K}$ is called a proper conformal vector field if $\Psi$ is a non-constant function. Accordingly, the following holds.

Proposition 4.2. If $M_{t}$ is null translating soliton in which $\bar{K}$ is conformal, then $\Psi=0$, that is; $\bar{K}$ is a killing vector field.

Proof. As $M_{t}$ is a null translating soliton, we have $\bar{K}=K+\mathbf{H}$, where $\mathbf{H}$ is the null mean curvature and given by $\mathbf{H}=\mathcal{S} N$. Then by simple calculations we have

$$
\begin{aligned}
\left(\mathcal{L}_{\bar{K}} \bar{g}\right)(X, Y) & =\left(\mathcal{L}_{K+\mathbf{H}} \bar{g}\right)(X, Y) \\
& =\left(\mathcal{L}_{K} g\right)(X, Y)+\bar{g}\left(\bar{\nabla}_{X} \mathbf{H}, Y\right)+\bar{g}\left(X, \bar{\nabla}_{Y} \mathbf{H}\right) \\
& =\left(\mathcal{L}_{K} g\right)(X, Y)+\mathcal{S} \bar{g}\left(\bar{\nabla}_{X} N, Y\right)+\mathcal{S} \bar{g}\left(X, \bar{\nabla}_{Y} N\right) \\
& +X(\mathcal{S}) \lambda(Y)+Y(\mathcal{S}) \lambda(X),
\end{aligned}
$$

for any $X, Y \in \Gamma(T M)$. Setting $X=X_{\alpha}, Y=X_{\beta}$ and the fact $\bar{K}$ is conformal, we get

$$
\begin{aligned}
\left(\mathcal{L}_{K} g\right)\left(X_{\alpha}, X_{\beta}\right) & =2 \Psi g\left(X_{\alpha}, X_{\beta}\right)-\mathcal{S} \bar{g}\left(\bar{\nabla}_{X_{\alpha}} N, X_{\beta}\right)-\mathcal{S} \bar{g}\left(X_{\alpha}, \bar{\nabla}_{X_{\beta}} N\right) \\
& =2 \Psi g\left(X_{\alpha}, X_{\beta}\right)+\mathcal{S} g\left(A_{N} X_{\alpha}, X_{\beta}\right)+\mathcal{S} g\left(X_{\alpha}, A_{N} X_{\beta}\right) \\
& =2 \Psi g\left(X_{\alpha}, X_{\beta}\right)+2 \mathcal{S} C\left(X_{\alpha}, X_{\beta}\right)
\end{aligned}
$$

in which we have used (2.4), (2.7) and symmetry of $C$. Then, using (4.4) and (4.6) we get

$$
\frac{\partial g_{\alpha \beta}}{\partial t}=-2 \Psi g_{\alpha \beta}-2 \mathcal{S} C_{\alpha \beta} .
$$

Comparing (4.7) and the evolution of $g$ in [22], we get $-2 \Psi g_{\alpha \beta}=0$, which implies that $\Psi=0$. Hence, $\bar{K}$ is killing and the proof is complete.

Observe that on a null translating soliton, (4.2) will imply that $\nabla_{X_{\alpha}} K_{\beta}=$ $\nabla_{X_{\beta}} K_{\alpha}$. Consequently, there is a function $\phi$ with $\nabla_{X_{\alpha}} \phi=K_{\alpha}$. Since the vector field is a gradient, we observe that null translating solitons are also gradient solitons [12], satisfying $\nabla_{X_{\alpha}} \nabla_{X_{\beta}} \phi=\mathcal{S} C_{\alpha \beta}$. Next, let us set

$$
\mathcal{B}_{\alpha \beta \gamma \mu}:=B_{\alpha \gamma} C_{\beta \mu} K_{\mu}+C_{\alpha \gamma} \nabla_{X_{\beta}} \mathcal{S}, \quad R_{\alpha \beta \gamma \mu}:=g\left(R\left(X_{\alpha}, X_{\beta}\right) X_{\gamma}, X_{\mu}\right),
$$


for $\alpha, \beta, \gamma, \mu \in\{1, \ldots, n\}$. Then, the following follows from the above discusion.

Proposition 4.3. On a null translating soliton, the following holds

$$
\nabla_{X_{\alpha}} \nabla_{X_{\beta}} \phi=\mathcal{S} C_{\alpha \beta} \quad \text { and } \quad \mathcal{B}_{\alpha \beta \gamma \mu}=\mathcal{B}_{\beta \alpha \gamma \mu} .
$$

Moreover, if $M$ is screen conformal and the local second fundamental form $B$ satisfy $B_{\alpha \beta}<\mathcal{S} g_{\alpha \beta}$, we have

$$
\nabla_{X_{\alpha}} \mathcal{S}+B_{\alpha \beta} K_{\beta}=0
$$

Proof. Differentiating the first relation of (4.8) we get

$$
\nabla_{X_{\alpha}} \nabla_{X_{\beta}} \nabla_{X_{\gamma}} \phi=C_{\beta \gamma} \nabla_{X_{\alpha}} \mathcal{S}+\mathcal{S} \nabla_{X_{\beta}} C_{\alpha \gamma}
$$

Inter-changing $X_{\alpha}$ and $X_{\beta}$ and subtracting the two equations, we have

$$
\nabla_{X_{\alpha}} \nabla_{X_{\beta}} \nabla_{X_{\gamma}} \phi-\nabla_{X_{\beta}} \nabla_{X_{\alpha}} \nabla_{X_{\gamma}} \phi=C_{\beta \gamma} \nabla_{X_{\alpha}} \mathcal{S}-C_{\alpha \gamma} \nabla_{X_{\beta}} \mathcal{S} .
$$

Then, from Gauss-Codazzi equation (3.7) of [7, p. 94], we have

$$
R_{\alpha \beta \gamma \mu}=B_{\alpha \gamma} C_{\beta \mu}-B_{\beta \gamma} C_{\alpha \mu} .
$$

From $(4.11),(4.12)$ and the fact $\nabla_{X_{\mu}} \phi=K_{\mu}$ we get

$$
B_{\alpha \gamma} C_{\beta \mu} K_{\mu}+C_{\alpha \gamma} \nabla_{X_{\beta}} \mathcal{S}=B_{\beta \gamma} C_{\alpha \mu} K_{\mu}+C_{\beta \gamma} \nabla_{X_{\alpha}} \mathcal{S}
$$

which proves the second relation of (4.8). Then when $M$ is screen conformal [7], we get

$$
B_{\alpha \gamma}\left(B_{\beta \mu} K_{\mu}+\nabla_{X_{\beta}} \mathcal{S}\right)=B_{\beta \gamma}\left(B_{\alpha \mu} K_{\mu}+\nabla_{X_{\alpha}} \mathcal{S}\right) .
$$

Setting $\mathcal{W}_{\alpha}:=B_{\alpha \mu} K_{\mu}+\nabla_{X_{\alpha}} \mathcal{S}$, such that (4.14) reduces to

$$
B_{\alpha \gamma} \mathcal{W}_{\beta}=B_{\beta \gamma} \mathcal{W}_{\alpha}
$$

Taking the trace on $\beta$ and $\gamma$ in (4.15) we obtain

$$
\left(\mathcal{S} g_{\alpha \beta}-B_{\alpha \beta}\right) \mathcal{W}_{\beta}=0
$$

Since $B_{\alpha \beta}<\mathcal{S} g_{\alpha \beta}$, then the matrix $\mathcal{S} g_{\alpha \beta}-B_{\alpha \beta}$ is strictly positive and hence, $\mathcal{W}_{\beta}=0$ and the proof is complete.

By the method of Hamiliton [12], we have the following differential Harnacktype inequality for null MCF. 
Theorem 4.4. Let $(M, g)$ be a null hypersurface of the Lorentzian manifold $(\bar{M}, \bar{g})$. If $M$ moves by null MCF, then

$$
\frac{\partial \mathcal{S}}{\partial t}+\frac{\mathcal{S}}{2 t}+2 \nabla \mathcal{S}(K)+B(K, P K) \geq 0
$$

for any tangent vector $K$, where $\mathcal{S}=\operatorname{tr}^{S}\left(A_{E}^{*}\right)$.

Proof. From [22] we have

$$
\frac{\partial \mathcal{S}}{\partial t}=\Delta^{s} \mathcal{S}+\mathcal{S} \operatorname{tr}^{s}\left(A_{E}^{*} \circ A_{N}\right)
$$

Now, differentiating the second relation of (4.2) and then applying the first relation of (4.2) and the fact $X_{\alpha} \cdot B_{\beta \gamma}=X_{\beta} \cdot B_{\alpha \gamma}$ around $p \in M$, we have

$$
\nabla_{E_{\alpha}} \nabla_{E_{\beta}} \mathcal{S}+K_{\gamma} \nabla_{E_{\gamma}} B_{\alpha \beta}+\mathcal{S} B_{\beta \gamma} C_{\gamma \alpha}=0 .
$$

Taking trace of (4.19) we get

$$
\Delta \mathcal{S}+K_{\gamma} \nabla_{E_{\gamma}} \mathcal{S}+\mathcal{S} \operatorname{tr}^{S}\left(A_{E}^{*} \circ A_{N}\right)=0
$$

Then substituting equation (4.20) in (4.18), we deduce that

$$
\frac{\partial \mathcal{S}}{\partial t}+K_{\alpha} \nabla_{E_{\alpha}} \mathcal{S}=0
$$

But from the second relation of (4.2), we have

$$
K_{\alpha} \nabla_{E_{\alpha}} \mathcal{S}+B_{\alpha \beta} K_{\alpha} K_{\beta}=0
$$

Adding (4.21) to (4.22), we deduce

$$
\frac{\partial \mathcal{S}}{\partial t}+2 \nabla \mathcal{S}(K)+B(P K, P K)=0 .
$$

Equation (4.23) is the basic differential null Harnack equation. As mentioned in [12], for solutions on $t>0$ we should really consider homothetically expanding solitons, which differ from the translating ones by terms that are basically lower order with a $\frac{1}{t}$ in them. This accounts for the existence of the term $\frac{1}{2 t} \mathcal{S}$ in our inequality, hence the proof.

Using Hamiliton's method [12] and Theorem 4.4 we state the following integrated version of null Hanarck inequality above, along paths in spacetime $\bar{M}^{n+2}$.

Theorem 4.5. For any weakly convex solution to the null mean curvature flow for $t>0$ we have

$$
\mathcal{S}\left(y_{2}, t_{2}\right) \geq \sqrt{\frac{t_{1}}{t_{2}}} e^{-\frac{1}{4} \Omega} \mathcal{S}\left(y_{1}, t_{1}\right)
$$


for any two points $y_{1}$ and $y_{2}$ on the evolving null hypersurface at times $t_{1}$ and $t_{2}$, with $T \geq t_{2}>t_{1}>0$, where

$$
\Omega:=\inf _{y} \int_{t_{1}}^{t_{2}} g\left(P \frac{d y}{d t}, P \frac{d y}{d t}\right) d t
$$

is the infimum over all paths $y(t)$ remaining on the null hypersurface at time $t$ with $\frac{d y}{d t}$ as the velocity vector of the path.

Proof. Along any path $y(t)=F(x(t), t)$ we have

$$
\frac{d \mathcal{S}}{d t}=\frac{\partial \mathcal{S}}{\partial t}+\nabla \mathcal{S}\left(\frac{d x}{d t}\right) .
$$

Using (4.24) and Theorem 4.4, with $K=\frac{1}{2} \frac{d x}{d t}$, we get

$$
\frac{d \mathcal{S}}{d t} \geq-\frac{1}{4} B\left(P \frac{d x}{d t}, P \frac{d x}{d t}\right)-\frac{1}{2 t} \mathcal{S} .
$$

But for a convex hypersurface, $B\left(P \frac{d x}{d t}, P \frac{d x}{d t}\right) \leq \mathcal{S} g\left(P \frac{d x}{d t}, P \frac{d x}{d t}\right)$ and hence (4.25) gives

$$
\frac{d \ln (\mathcal{S})}{d t} \geq-\frac{1}{4} g\left(P \frac{d x}{d t}, P \frac{d x}{d t}\right)-\frac{1}{2 t}
$$

where $P \frac{d x}{d t}$ is the screen component of $\frac{d y}{d t}$, such that (4.26) leads to

$$
\log \left(\frac{\mathcal{S}\left(y_{2}, t_{2}\right)}{\mathcal{S}\left(y_{1}, t_{1}\right)}\right) \geq-\frac{1}{2} \log \left(\frac{t_{2}}{t_{1}}\right)-\frac{1}{4} \Omega
$$

where $\Omega:=\inf _{y} \int_{t_{1}}^{t_{2}} g\left(P \frac{d y}{d t}, P \frac{d y}{d t}\right) d t$, and the result follows by exponentiating, which completes the proof.

\section{Acknowledgments}

This work is based on the research supported wholly / in part by the National Research Foundation of South Africa (Grant Numbers: 95931 and 106072).

\section{References}

[1] A. Ashtekar and B. Krishnan, Dynamical horizons and their properties, Physical Review D, 68 (2003) 14030-10455.

[2] B. Andrews, Monotone quantities and unique limits for evolving convex hypersurfaces, Internat. Math. Res. Notices 1997 (1997), 1001-1031.

[3] S. Aretakis, Lecture notes on general relativity, https://web.math.princeton.edu/ aretakis/columbiaGR.pdf.

[4] C. Atindogbé, Scalar curvature on lightlike hypersurfaces, Balkan Society of Geometers, Geometry Balkan Press 2009, Applied Sciences, 11 (2009) 9-18. 
[5] C. Atindogbé, J.-P. Ezin and J. Tossa, Pseudo-inversion of degenerate metrics Int. J. Math. Math. Sci., 55 (2003) 3479-3501.

[6] R. C. Baker, The mean curvature flow of submanifolds of high codimension, $\mathrm{PhD}$ thesis, Australian National University, 2010.

[7] K. L. Duggal and A. Bejancu. Lightlike submanifolds of semi-Riemannian manifolds and Applications. Mathematics and its Applications ( Kluwer Academic Publishers, 1996).

[8] K. L. Duggal and B. Sahin, Differential geometry of lightlike submanifolds (Frontiers in Mathematics, Birkhäuser Verlag, Basel, 2010).

[9] M. Gage, and R. Hamilton, The heat equation shrinking convex plane curves, J. Differential Geom. 23 no. 1 (1986) 69-96.

[10] L. Guanghan, T. Daping and W. Chuanxi, Translating solitons of mean curvature flow of noncompact submanifolds, Math Phys Anal Geom. 14 (2011) 83-99.

[11] M. Guti'errez and B. Olea. Induced Riemannian structures on null hypersurfaces. Math. Nachr. 289 (2016) 1219-1236.

[12] R. S. Hamilton, Harnack estimate for the mean curvature flow, J. Differential Geom. 41 (1995), 215-226.

[13] G. Huisken, Asymptotic behaviour for singularities of the mean curvature flow, J. Diff. Geom. 31 (1990) 285-299 .

[14] G. Huisken, Flow by mean curvature of convex surfaces into spheres, J. Differential Geometry 20 (1984) 237-266.

[15] G. Huisken, Evolution of hypersurfaces by their curvature in Riemannian manifolds. Proceedings of the International Congress of Mathematicians, Doc. Math. 1998, Extra Vol. II, 349-360.

[16] G. Huisken and C. Sinestrari, Mean curvature flow singularities for mean convex surfaces. Calc. Var. Partial Differential Equations, 8 (1) (1999), 1-14.

[17] D. H. Jin, Ascreen lightlike hypersurfaces of an indefinite Sasakian manifold. J. Korean Soc. Math. Educ. Ser. B: Pure Appl. Math., 20 no. 1 (2013) 25-35.

[18] C. Mantegazza, Lecture notes on mean curvature flow, Progress in Mathematics, 290 (2012).

[19] B. O'Neill, Semi-Riemannian Geometry, with Applications to Relativity (New York: Academic Press 1983).

[20] M. Ritoré, C. Sinestrari, Mean curvature flow and isoperimetric inequalities, Advanced Courses in Mathematics. CRM Barcelona. Birkhauser Verlag, Basel, 2010.

[21] W. Sheng and X. Wang, Regularity and Singularity in the Mean Curvature Flow, Trends in Partial Differential Equations, ALM 10, pp. 399-436.

[22] S. Ssekajja and F. Massamba, Null hypersurfaces evolved by their mean curvatures in Lorentzian manifold, Preprint.

[23] M. E. Taylor, Partial Differential Equations III: Nonlinear Equations, ( 117, Applied Mathematical Sciences, Springer, 1996). 
1 School of Mathematics, Statistics and Computer Science University of KwaZulu-Natal

Private Bag X01, Scottsville 3209

South Africa

E-mail address: ssekajja.samuel.buwaga@aims-senegal.org

2 School of Mathematics, Statistics and Computer Science University of KwaZulu-Natal

Private Bag X01, Scottsville 3209

South Africa

E-mail address: massfort@yahoo.fr, Massamba@ukzn.ac.za 